\title{
DISPERSIVE OPTICAL SOLITONS WITH SCHRÖDINGER-HIROTA EQUATION BY EXTENDED TRIAL EQUATION METHOD
}

\author{
Mehmet Ekici ${ }^{1}$, Mohammad Mirzazadeh ${ }^{2}$, Abdullah Sonmezoglu ${ }^{1}$, Malik Zaka Ullah ${ }^{3}$, \\ Mir Asma ${ }^{4}$, Qin Zhou ${ }^{5}$, Seithuti P. Moshokoa ${ }^{6}$, Anjan Biswas ${ }^{3,6}$ \& Milivoj Belic ${ }^{7}$ \\ ${ }^{1}$ Department of Mathematics, Faculty of Science and Arts, \\ Bozok University, 66100 Yozgat, Turkey \\ ${ }^{2}$ Department of Engineering Sciences, Faculty of Technology and Engineering, \\ East of Guilan, University of Guilan, P.C. 44891-63157, Rudsar-Vajargah, Iran \\ ${ }^{3}$ Department of Mathematics, Faculty of Science, \\ King Khalid University, PO Box 9004, Abha-61413, Saudi Arabia \\ ${ }^{4}$ Institute of Mathematical Sciences, Faculty of Science, \\ University of Malaya, 50601 Kuala Lumpur, Malaysia \\ 5 School of Electronics and Information Engineering, \\ Wuhan Donghu University, Wuhan, 430212, PR China \\ ${ }^{6}$ Department of Mathematics and Statistics, \\ Tshwane University of Technology, Pretoria-0008, South Africa \\ 7 Science Program, Texas A \& M University at Qatar, \\ PO Box 23874, Doha, Qatar
}

\begin{abstract}
This paper obtains bright, dark and singular soliton solutions from perturbed Schrödinger-Hirota equation that governs the propagation of dispersive pulses through optical fibers. The trial equation method is adopted to achieve this goal. Both Kerr and power laws of nonlinearity are covered.
\end{abstract}

Key words: solitons; dispersion; Schrödinger-Hirota equation; extended trial equation method. 


\section{INTRODUCTION}

Optical solitons serve as an amazing jewel in the field of Nonlinear Optics. These soliton molecules form the basic fabric in modern telecommunication industry [1-15]. However, when higher order dispersion, such as third order dispersion (3OD), kicks in, the governing model is Schrödinbger-Hirota equation (SHE) as opposed to the usual nonlinear Schrödinger's equation (NLSE). It is Lie transform that converts NLSE with 3OD to SHE. In addition to the usual group velocity dispersion (GVD), this paper includes spatio-temporal dispersion (STD) that makes the model well-posed as pointed out during $2012[4,5]$.

The extended trial equation method is the integration scheme employed in this paper to extract the soliton solutions to SHE with Hamiltonian type petrturbations. Both Kerr and power laws of nonlinearity are studied. Bright, dark and singular soliton solutions are obtained for the governing equation. As a byproduct, plane waves, periodic singular waves and solutions in terms of Jacobi elliptic functions are presented.

\section{GOVERNING EQUATION}

The nonlinear evolution equation that models the propagation of solitons through optical fibers, with 3OD is the NLSE

$$
i u_{t}+\frac{1}{2} u_{x x}+|u|^{2} u=-i \lambda u_{x x x}
$$

Here $\lambda$ is the coefficient of 3OD. The first term on the left hand side is the temporal evolution, while the second term represents GVD. Also, on left side, the third term comes with Kerr law nonlinearity. The inclusion of 3OD is meaningful when GVD is low. The following Lie symmetry is introduced to analyze (1) further.

$$
q=u-3 i \lambda\left[u_{x}+2 u \int_{-\infty}^{x}|u(\xi)|^{2} d \xi\right] .
$$

which transforms (1) to

$$
i q_{t}+\frac{1}{2} q_{x x}+|q|^{2} q+i \lambda\left(q_{x x x}+6|q|^{2} q_{x}\right)=0,
$$

after neglecting higher order terms [1-3]. Equation (3) is SHE with Kerr law nonlinearity. Therefore, SHE models transmission of dispersive optical solitons through nonlinear fibers. With arbitrary coefficients, SHE can be rewritten as

$$
i q_{t}+a q_{x x}+c|q|^{2} q+i\left(\gamma q_{x x x}+\sigma|q|^{2} q_{x}\right)=0 .
$$

Physically $\sigma$ represents nonlinear dispersion. It was indicated during 2012, that GVD alone makes the governing model ill-posed $[4,5]$. Therefore, it was proposed that inclusion of an additional dispersion term, namely the spatiotemporal dispersion (STD) introduces well-posedness $[4,5]$. Hence, SHE with STD is

$$
i q_{t}+a q_{x x}+b q_{x t}+c|q|^{2} q+i\left(\gamma q_{x x x}+\sigma|q|^{2} q_{x}\right)=0
$$

where the coefficient of $b$ represents STD. Finally, with perturbation terms, SHE with STD extends to

$$
i q_{t}+a q_{x x}+b q_{x t}+c|q|^{2} q+i\left(\gamma q_{x x x}+\sigma|q|^{2} q_{x}\right)=i \alpha q_{x}+i \lambda\left(|q|^{2} q\right)_{x}+i \nu\left(|q|^{2}\right)_{x} q .
$$

In (6), $\lambda$ and $\nu$ are self-steepening and nonlinear dispersion respectively. This paper will carry out the integration of the perturbed SHE with STD by extended trial equation method [6-10]. This analysis is detailed in next section. 


\section{SOLITON SOLUTIONS}

For the traveling wave solution to (6), the starting hypothesis is given by [11, 12]

$$
q(x, t)=g(\xi) e^{i \phi(x, t)},
$$

where in $(7), g(x, t)$ represents the wave profile and $\phi(x, t)$ is the phase component of the soliton that is defined as

$$
\phi(x, t)=-\kappa x+\omega t+\theta
$$

and also

$$
\xi=x-v t
$$

Here, in (9) $v$ represents the soliton velocity, while from phase component that is given by (8), $\kappa$ is the constant soliton frequency, $\omega$ is soliton wave number that is also taken to be constant and $\theta$ is the phase constant for the soliton. The study will now be subdivided into two subsections that concentrates on Kerr law and power law media.

\subsection{KERR LAW}

Substituting (7) into (6), and decomposing into real and imaginary parts lead to [11, 12]

$$
(a-b v \omega+3 \gamma \kappa) g^{\prime \prime}=\left(\omega+b \omega \kappa+a \kappa^{2}+\gamma \kappa^{3}+\alpha \kappa\right) g-(\lambda \kappa-\sigma \kappa-c) g^{3},
$$

and

$$
\gamma g^{\prime \prime \prime}=\left\{v+\alpha+2 a \kappa+b \omega(\omega+v \kappa)+3 \gamma \kappa^{2}\right\} g^{\prime}-(\sigma-3 \lambda-2 \nu) g^{2} g^{\prime},
$$

respectively, after simplification. The notations $g^{\prime}$ and $g^{\prime \prime}$ are given by $g^{\prime}=d g / d \xi, g^{\prime \prime}=d^{2} g / d \xi^{2}$.

From the linearly independent functions, it shows that third-order dispersion must vanish, namely

$$
\gamma=0
$$

Eq. (12) shows that soliton solutions for Kerr law nonlinearity, will exist provided third order dispersion vanishes. This observation is being made for the first time in this paper. Therefore, Eqs. (10) and (11), by virtue of Eq. (12), reduces to

$$
(a-b v \omega) g^{\prime \prime}-\left(\omega+b \omega \kappa+a \kappa^{2}+\alpha \kappa\right) g+(\lambda \kappa-\sigma \kappa-c) g^{3}=0
$$

and

$$
\{v+\alpha+2 a \kappa+b \omega(\omega+v \kappa)\} g^{\prime}-(\sigma-3 \lambda-2 \nu) g^{2} g^{\prime}=0,
$$

respectively. The remaining linearly independent functions, from Eq. (11), lead to the constraint

$$
\sigma-3 \lambda-2 \nu=0
$$

and the speed of the soliton

$$
v=-\frac{\alpha+2 a \kappa+b \omega^{2}}{1+b \omega \kappa} .
$$

We will now analyze Eq. (13) to secure soliton solutions by extended trial equation method. To start off with the solution of Eq. (13), the following assumption for the soliton structure is made

$$
g=\sum_{i=0}^{\varsigma} \tau_{i} \Psi^{i}
$$


where

$$
\left(\Psi^{\prime}\right)^{2}=\Lambda(\Psi)=\frac{\Phi(\Psi)}{\Upsilon(\Psi)}=\frac{\mu_{\varrho} \Psi^{\varrho}+\cdots+\mu_{1} \Psi+\mu_{0}}{\chi_{\rho} \Psi^{\rho}+\cdots+\chi_{1} \Psi+\chi_{0}} .
$$

Using (17) along with (18), we have the terms $\left(g^{\prime}\right)^{2}$ and $g^{\prime \prime}$ as follows:

$$
\left(g^{\prime}\right)^{2}=\frac{\Phi(\Psi)}{\Upsilon(\Psi)}\left(\sum_{i=0}^{\varsigma} i \tau_{i} \Psi^{i-1}\right)^{2},
$$

and

$$
g^{\prime \prime}=\frac{\Phi^{\prime}(\Psi) \Upsilon(\Psi)-\Phi(\Psi) \Upsilon^{\prime}(\Psi)}{2 \Upsilon^{2}(\Psi)}\left(\sum_{i=0}^{\varsigma} i \tau_{i} \Psi^{i-1}\right)+\frac{\Phi(\Psi)}{\Upsilon(\Psi)}\left(\sum_{i=0}^{\varsigma} i(i-1) \tau_{i} \Psi^{i-2}\right),
$$

where $\Phi(\Psi)$ and $\Upsilon(\Psi)$ are polynomials of $\Psi$. We can reduce Eq. (18) to the elementary integral form as follows:

$$
\pm\left(\xi-\xi_{0}\right)=\int \frac{d \Psi}{\sqrt{\Lambda(\Psi)}}=\int \sqrt{\frac{\Upsilon(\Psi)}{\Phi(\Psi)}} d \Psi .
$$

Using the balance principle, we find that

$$
\varrho=\rho+2 \varsigma+2 .
$$

When $\varrho=4, \rho=0$ and $\varsigma=1$ in Eq. (22), we obtain

$$
\begin{gathered}
g=\tau_{0}+\tau_{1} \Psi, \\
g^{\prime \prime}=\frac{\tau_{1}\left(4 \mu_{4} \Psi^{3}+3 \mu_{3} \Psi^{2}+2 \mu_{2} \Psi+\mu_{1}\right)}{2 \chi_{0}},
\end{gathered}
$$

where $\mu_{4} \neq 0$ and $\chi_{0} \neq 0$. Substituting Eqs. (23) and (24) into Eq. (13), collecting the coefficients of $\Psi$, and solving the resulting system, we find the following results:

$$
\begin{aligned}
& \mu_{2}=\frac{\mu_{1} \tau_{1}\left[a+b \kappa(a+v \alpha)+a b v \kappa^{2}+3 b v \tau_{0}^{2}(c-\kappa \lambda+\kappa \sigma)\right]+4 \tau_{0}^{3} \chi_{0}(1+b \kappa)[c+\kappa(\sigma-\lambda)]}{2 \tau_{0}\left[a+b \kappa(a+v \alpha)+a b v \kappa^{2}+b v \tau_{0}^{2}(c+\kappa(\sigma-\lambda))\right]}, \\
& \mu_{3}=\frac{\tau_{1}[c+\kappa(\sigma-\lambda)]\left[b v \mu_{1} \tau_{1}+2 \tau_{0} \chi_{0}(1+b \kappa)\right]}{a+b \kappa(a+v \alpha)+a b v \kappa^{2}+b v \tau_{0}^{2}[c+\kappa(\sigma-\lambda)]}, \\
& \mu_{4}=\frac{\tau_{1}^{2}[c+\kappa(\sigma-\lambda)]\left[b v \mu_{1} \tau_{1}+2 \tau_{0} \chi_{0}(1+b \kappa)\right]}{4 \tau_{0}\left[a+b \kappa(a+v \alpha)+a b v \kappa^{2}+b v \tau_{0}^{2}(c+\kappa(\sigma-\lambda))\right]}, \\
& \omega=\frac{a \mu_{1} \tau_{1}-2 \tau_{0} \chi_{0}\left[\kappa(\alpha+a \kappa)+\tau_{0}^{2}(c-\kappa \lambda+\kappa \sigma)\right]}{b v \mu_{1} \tau_{1}+2 \tau_{0} \chi_{0}(1+b \kappa)}, \\
& \mu_{0}=\mu_{0}, \quad \mu_{1}=\mu_{1}, \quad \chi_{0}=\chi_{0}, \quad \tau_{0}=\tau_{0}, \quad \tau_{1}=\tau_{1} .
\end{aligned}
$$

Substituting these results into Eqs. (18) and (21), we find that

$$
\pm\left(\xi-\xi_{0}\right)=W \int \frac{d \Psi}{\sqrt{\Lambda(\Psi)}}
$$

where

$$
\Lambda(\Psi)=\Psi^{4}+\frac{\mu_{3}}{\mu_{4}} \Psi^{3}+\frac{\mu_{2}}{\mu_{4}} \Psi^{2}+\frac{\mu_{1}}{\mu_{4}} \Psi+\frac{\mu_{0}}{\mu_{4}}, \quad W=\sqrt{\frac{\chi_{0}}{\mu_{4}}} .
$$

Consequently, one recovers the traveling wave solutions to the perturbed SHE with STD (6) as the following: 
When $\Lambda(\Psi)=\left(\Psi-\lambda_{1}\right)^{4}$,

$$
\begin{aligned}
q(x, t)= & \left\{\tau_{0}+\tau_{1} \lambda_{1} \pm \frac{\tau_{1} W}{x+\left(\frac{\alpha+2 a \kappa+b \omega^{2}}{1+b \omega \kappa}\right) t-\xi_{0}}\right\} \\
& \times \exp \left[i\left\{-\kappa x+\left(\frac{a \mu_{1} \tau_{1}-2 \tau_{0} \chi_{0}\left[\kappa(\alpha+a \kappa)+\tau_{0}^{2}(c-\kappa \lambda+\kappa \sigma)\right]}{b v \mu_{1} \tau_{1}+2 \tau_{0} \chi_{0}(1+b \kappa)}\right) t+\theta\right\}\right] .
\end{aligned}
$$

When $\Lambda(\Psi)=\left(\Psi-\lambda_{1}\right)^{3}\left(\Psi-\lambda_{2}\right)$ and $\lambda_{2}>\lambda_{1}$,

$$
\begin{aligned}
q(x, t)= & \left\{\tau_{0}+\tau_{1} \lambda_{1}+\frac{4 W^{2}\left(\lambda_{2}-\lambda_{1}\right) \tau_{1}}{4 W^{2}-\left[\left(\lambda_{1}-\lambda_{2}\right)\left(x+\left(\frac{\alpha+2 a \kappa+b \omega^{2}}{1+b \omega \kappa}\right) t-\xi_{0}\right)\right]^{2}}\right\} \\
& \times \exp \left[i\left\{-\kappa x+\left(\frac{a \mu_{1} \tau_{1}-2 \tau_{0} \chi_{0}\left[\kappa(\alpha+a \kappa)+\tau_{0}^{2}(c-\kappa \lambda+\kappa \sigma)\right]}{b v \mu_{1} \tau_{1}+2 \tau_{0} \chi_{0}(1+b \kappa)}\right) t+\theta\right\}\right] .
\end{aligned}
$$

When $\Lambda(\Psi)=\left(\Psi-\lambda_{1}\right)^{2}\left(\Psi-\lambda_{2}\right)^{2}$,

$$
\begin{aligned}
q(x, t)= & \left\{\tau_{0}+\tau_{1} \lambda_{2}+\frac{\left(\lambda_{2}-\lambda_{1}\right) \tau_{1}}{\exp \left[\frac{\lambda_{1}-\lambda_{2}}{W}\left(x+\left(\frac{\alpha+2 a \kappa+b \omega^{2}}{1+b \omega \kappa}\right) t-\xi_{0}\right)\right]-1}\right\} \\
& \times \exp \left[i\left\{-\kappa x+\left(\frac{a \mu_{1} \tau_{1}-2 \tau_{0} \chi_{0}\left[\kappa(\alpha+a \kappa)+\tau_{0}^{2}(c-\kappa \lambda+\kappa \sigma)\right]}{b v \mu_{1} \tau_{1}+2 \tau_{0} \chi_{0}(1+b \kappa)}\right) t+\theta\right\}\right],
\end{aligned}
$$

and

$$
\begin{aligned}
q(x, t)= & \left\{\tau_{0}+\tau_{1} \lambda_{1}+\frac{\left(\lambda_{1}-\lambda_{2}\right) \tau_{1}}{\exp \left[\frac{\lambda_{1}-\lambda_{2}}{W}\left(x+\left(\frac{\alpha+2 a \kappa+b \omega^{2}}{1+b \omega \kappa}\right) t-\xi_{0}\right)\right]-1}\right\} \\
& \times \exp \left[i\left\{-\kappa x+\left(\frac{a \mu_{1} \tau_{1}-2 \tau_{0} \chi_{0}\left[\kappa(\alpha+a \kappa)+\tau_{0}^{2}(c-\kappa \lambda+\kappa \sigma)\right]}{b v \mu_{1} \tau_{1}+2 \tau_{0} \chi_{0}(1+b \kappa)}\right) t+\theta\right\}\right] .
\end{aligned}
$$

When $\Lambda(\Psi)=\left(\Psi-\lambda_{1}\right)^{2}\left(\Psi-\lambda_{2}\right)\left(\Psi-\lambda_{3}\right)$ and $\lambda_{1}>\lambda_{2}>\lambda_{3}$,

$$
\begin{aligned}
q(x, t)= & \left\{\tau_{0}+\tau_{1} \lambda_{1}-\frac{2\left(\lambda_{1}-\lambda_{2}\right)\left(\lambda_{1}-\lambda_{3}\right) \tau_{1}}{2 \lambda_{1}-\lambda_{2}-\lambda_{3}+\left(\lambda_{3}-\lambda_{2}\right) \cosh \left(\frac{\sqrt{\left(\lambda_{1}-\lambda_{2}\right)\left(\lambda_{1}-\lambda_{3}\right)}}{W}\left[x+\left(\frac{\alpha+2 a \kappa+b \omega^{2}}{1+b \omega \kappa}\right) t\right]\right)}\right\} \\
& \times \exp \left[i\left\{-\kappa x+\left(\frac{a \mu_{1} \tau_{1}-2 \tau_{0} \chi_{0}\left[\kappa(\alpha+a \kappa)+\tau_{0}^{2}(c-\kappa \lambda+\kappa \sigma)\right]}{b v \mu_{1} \tau_{1}+2 \tau_{0} \chi_{0}(1+b \kappa)}\right) t+\theta\right\}\right] .
\end{aligned}
$$

When $\Lambda(\Psi)=\left(\Psi-\lambda_{1}\right)\left(\Psi-\lambda_{2}\right)\left(\Psi-\lambda_{3}\right)\left(\Psi-\lambda_{4}\right)$ and $\lambda_{1}>\lambda_{2}>\lambda_{3}>\lambda_{4}$,

$$
\begin{aligned}
q(x, t)= & \left\{\tau_{0}+\tau_{1} \lambda_{2}+\frac{\tau_{1}\left(\lambda_{1}-\lambda_{2}\right)\left(\lambda_{4}-\lambda_{2}\right)}{\lambda_{4}-\lambda_{2}+\left(\lambda_{1}-\lambda_{4}\right) \operatorname{sn}^{2}\left[ \pm \frac{\sqrt{\left(\lambda_{1}-\lambda_{3}\right)\left(\lambda_{2}-\lambda_{4}\right)}}{2 W}\left[x+\left(\frac{\alpha+2 a \kappa+b \omega^{2}}{1+b \omega \kappa}\right) t-\xi_{0}\right], l\right]}\right\} \\
& \times \exp \left[i\left\{-\kappa x+\left(\frac{a \mu_{1} \tau_{1}-2 \tau_{0} \chi_{0}\left[\kappa(\alpha+a \kappa)+\tau_{0}^{2}(c-\kappa \lambda+\kappa \sigma)\right]}{b v \mu_{1} \tau_{1}+2 \tau_{0} \chi_{0}(1+b \kappa)}\right) t+\theta\right\}\right],
\end{aligned}
$$


where

$$
l^{2}=\frac{\left(\lambda_{2}-\lambda_{3}\right)\left(\lambda_{1}-\lambda_{4}\right)}{\left(\lambda_{1}-\lambda_{3}\right)\left(\lambda_{2}-\lambda_{4}\right)} .
$$

Note that $\lambda_{i}(i=1, \ldots, 4)$ are the roots of the polynomial equation

$$
\Lambda(\Psi)=0 .
$$

When $\tau_{0}=-\tau_{1} \lambda_{1}$ and $\xi_{0}=0$, solutions (28)-(32) can be reduced to plane wave solutions

$$
\begin{aligned}
q(x, t)= & \left\{ \pm \frac{\tau_{1} W}{x+\left(\frac{\alpha+2 a \kappa+b \omega^{2}}{1+b \omega \kappa}\right) t}\right\} \\
& \times \exp \left[i\left\{-\kappa x+\left(\frac{a \mu_{1} \tau_{1}-2 \tau_{0} \chi_{0}\left[\kappa(\alpha+a \kappa)+\tau_{0}^{2}(c-\kappa \lambda+\kappa \sigma)\right]}{b v \mu_{1} \tau_{1}+2 \tau_{0} \chi_{0}(1+b \kappa)}\right) t+\theta\right\}\right], \\
q(x, t)= & \left\{\frac{4 W^{2}\left(\lambda_{2}-\lambda_{1}\right) \tau_{1}}{4 W^{2}-\left[\left(\lambda_{1}-\lambda_{2}\right)\left(x+\left(\frac{\alpha+2 a \kappa+b \omega^{2}}{1+b \omega \kappa}\right) t\right)\right]^{2}}\right\} \\
& \times \exp \left[i\left\{-\kappa x+\left(\frac{a \mu_{1} \tau_{1}-2 \tau_{0} \chi_{0}\left[\kappa(\alpha+a \kappa)+\tau_{0}^{2}(c-\kappa \lambda+\kappa \sigma)\right]}{b v \mu_{1} \tau_{1}+2 \tau_{0} \chi_{0}(1+b \kappa)}\right) t+\theta\right\}\right],
\end{aligned}
$$

singular soliton solutions

$$
\begin{aligned}
q(x, t)= & \frac{\left(\lambda_{2}-\lambda_{1}\right) \tau_{1}}{2}\left\{1 \mp \operatorname{coth}\left[\frac{\lambda_{1}-\lambda_{2}}{2 W}\left(x+\left(\frac{\alpha+2 a \kappa+b \omega^{2}}{1+b \omega \kappa}\right) t\right)\right]\right\} \\
& \times \exp \left[i\left\{-\kappa x+\left(\frac{a \mu_{1} \tau_{1}-2 \tau_{0} \chi_{0}\left[\kappa(\alpha+a \kappa)+\tau_{0}^{2}(c-\kappa \lambda+\kappa \sigma)\right]}{b v \mu_{1} \tau_{1}+2 \tau_{0} \chi_{0}(1+b \kappa)}\right) t+\theta\right\}\right],
\end{aligned}
$$

and bright soliton solution

$$
\begin{aligned}
q(x, t)= & \left\{\frac{A}{C+\cosh \left[B\left(x+\left(\frac{\alpha+2 a \kappa+b \omega^{2}}{1+b \omega \kappa}\right) t\right)\right]}\right\} \\
& \times \exp \left[i\left\{-\kappa x+\left(\frac{a \mu_{1} \tau_{1}-2 \tau_{0} \chi_{0}\left[\kappa(\alpha+a \kappa)+\tau_{0}^{2}(c-\kappa \lambda+\kappa \sigma)\right]}{b v \mu_{1} \tau_{1}+2 \tau_{0} \chi_{0}(1+b \kappa)}\right) t+\theta\right\}\right],
\end{aligned}
$$

where

$$
A=\frac{2\left(\lambda_{1}-\lambda_{2}\right)\left(\lambda_{1}-\lambda_{3}\right) \tau_{1}}{\lambda_{3}-\lambda_{2}}, \quad B=\frac{\sqrt{\left(\lambda_{1}-\lambda_{2}\right)\left(\lambda_{1}-\lambda_{3}\right)}}{W}, \quad C=\frac{2 \lambda_{1}-\lambda_{2}-\lambda_{3}}{\lambda_{3}-\lambda_{2}} .
$$

Here, $A$ is the amplitude of the soliton, while $B$ is the inverse width of the soliton. These solitons exist for $\tau_{1}<0$. Moreover, when $\tau_{0}=-\tau_{1} \lambda_{2}$ and $\xi_{0}=0$, Jacobi elliptic function solution (33) can be written as

$$
\begin{aligned}
q(x, t)= & \left\{\frac{A_{1}}{C_{1}+\operatorname{sn}^{2}\left[B_{j}\left(x+\left(\frac{\alpha+2 a \kappa+b \omega^{2}}{1+b \omega \kappa}\right) t\right), \frac{\left(\lambda_{2}-\lambda_{3}\right)\left(\lambda_{1}-\lambda_{4}\right)}{\left(\lambda_{1}-\lambda_{3}\right)\left(\lambda_{2}-\lambda_{4}\right)}\right]}\right\} \\
& \times \exp \left[i\left\{-\kappa x+\left(\frac{a \mu_{1} \tau_{1}-2 \tau_{0} \chi_{0}\left[\kappa(\alpha+a \kappa)+\tau_{0}^{2}(c-\kappa \lambda+\kappa \sigma)\right]}{b v \mu_{1} \tau_{1}+2 \tau_{0} \chi_{0}(1+b \kappa)}\right) t+\theta\right\}\right],
\end{aligned}
$$


where

$$
\begin{gathered}
A_{1}=\frac{\tau_{1}\left(\lambda_{1}-\lambda_{2}\right)\left(\lambda_{4}-\lambda_{2}\right)}{\lambda_{1}-\lambda_{4}}, \quad C_{1}=\frac{\lambda_{4}-\lambda_{2}}{\lambda_{1}-\lambda_{4}}, \\
B_{j}=\frac{(-1)^{j} \sqrt{\left(\lambda_{1}-\lambda_{3}\right)\left(\lambda_{2}-\lambda_{4}\right)}}{2 W} \text { for } \mathrm{j}=1,2 .
\end{gathered}
$$

Remark-1: When the modulus $l \rightarrow 1$, a second form of singular optical soliton solutions emerge as below:

$$
\begin{aligned}
q(x, t)= & \left\{\frac{A_{1}}{C_{1}+\tanh ^{2}\left[B_{j}\left(x+\left(\frac{\alpha+2 a \kappa+b \omega^{2}}{1+b \omega \kappa}\right) t\right)\right]}\right\} \\
& \times \exp \left[i\left\{-\kappa x+\left(\frac{a \mu_{1} \tau_{1}-2 \tau_{0} \chi_{0}\left[\kappa(\alpha+a \kappa)+\tau_{0}^{2}(c-\kappa \lambda+\kappa \sigma)\right]}{b v \mu_{1} \tau_{1}+2 \tau_{0} \chi_{0}(1+b \kappa)}\right) t+\theta\right\}\right],
\end{aligned}
$$

where $\lambda_{3}=\lambda_{4}$.

Remark-2: However, if $l \rightarrow 0$, periodic singular solutions fall out as follows:

$$
\begin{aligned}
q(x, t)= & \left\{\frac{A_{1}}{C_{1}+\sin ^{2}\left[B_{j}\left(x+\left(\frac{\alpha+2 a \kappa+b \omega^{2}}{1+b \omega \kappa}\right) t\right)\right]}\right\} \\
& \times \exp \left[i\left\{-\kappa x+\left(\frac{a \mu_{1} \tau_{1}-2 \tau_{0} \chi_{0}\left[\kappa(\alpha+a \kappa)+\tau_{0}^{2}(c-\kappa \lambda+\kappa \sigma)\right]}{b v \mu_{1} \tau_{1}+2 \tau_{0} \chi_{0}(1+b \kappa)}\right) t+\theta\right\}\right],
\end{aligned}
$$

where $\lambda_{2}=\lambda_{3}$.

\subsection{POWER LAW}

For power law nonlinearity, perturbed SHE with STD extends to

$$
i q_{t}+a q_{x x}+b q_{x t}+c|q|^{2 n} q+i\left(\gamma q_{x x x}+\sigma|q|^{2 n} q_{x}\right)=i \alpha q_{x}+i \lambda\left(|q|^{2 n} q\right)_{x}+i \nu\left(|q|^{2 n}\right)_{x} q,
$$

where the power-law nonlinearity factor $n$ kicks in. It was proved earlier that the restriction $0<n<2$ must remain valid to avoid self-focusing singularity [13]. It is clear that Kerr law nonlinearity falls out upon setting $n=1$ in (46). In order to integrate (46), the same hypothesis given by (7)-(9) is substituted into (46) and subsequently decomposed into real and imaginary parts. These respectively lead to

$$
(a-b v \omega+3 \gamma \kappa) g^{\prime \prime}=\left(\omega+b \omega \kappa+a \kappa^{2}+\gamma \kappa^{3}+\alpha \kappa\right) g-(\lambda \kappa-\sigma \kappa-c) g^{2 n+1},
$$

and

$$
\gamma g^{\prime \prime \prime}=\left\{v+\alpha+2 a \kappa+b \omega(\omega+v \kappa)+3 \gamma \kappa^{2}\right\} g^{\prime}-(\sigma-3 \lambda-2 \nu) g^{2 n} g^{\prime},
$$

after simplification. From (48), once again $\gamma=0$. The speed $v$ of the soliton from the linearly independent functions is also given by (16). The constraint condition (15) also fall out from these linearly independent functions. Consequently, the real part Eq. (47) changes to

$$
(a-b v \omega) g^{\prime \prime}=\left(\omega+b \omega \kappa+a \kappa^{2}+\alpha \kappa\right) g-(\lambda \kappa-\sigma \kappa-c) g^{2 n+1} .
$$

Balancing $g^{\prime \prime}$ with $g^{2 n+1}$ gives $N=\frac{1}{n}$. In order to obtain closed form solutions, we use the transformation

$$
g=U^{\frac{1}{2 n}}
$$


that will reduce Eq. (49) into the ODE

$$
(a-b v \omega)\left\{(1-2 n)\left(U^{\prime}\right)^{2}+2 n U U^{\prime \prime}\right\}=4 n^{2}\left(\omega+b \omega \kappa+a \kappa^{2}+\alpha \kappa\right) U^{2}-4 n^{2}(\lambda \kappa-\sigma \kappa-c) U^{3} .
$$

We will now analyze Eq. (51) to construct soliton and other solutions by extended trial equation method. Using the balance principle, we recover that

$$
\varrho=\rho+\varsigma+2 .
$$

Case-1: When $\varrho=3, \rho=0$ and $\varsigma=1$ in Eq. (52), we have

$$
\begin{gathered}
U=\tau_{0}+\tau_{1} \Psi, \\
\left(U^{\prime}\right)^{2}=\frac{\tau_{1}^{2}\left(\mu_{3} \Psi^{3}+\mu_{2} \Psi^{2}+\mu_{1} \Psi+\mu_{0}\right)}{\chi_{0}}, \\
U^{\prime \prime}=\frac{\tau_{1}\left(3 \mu_{3} \Psi^{2}+2 \mu_{2} \Psi+\mu_{1}\right)}{2 \chi_{0}},
\end{gathered}
$$

where $\mu_{3} \neq 0$ and $\chi_{0} \neq 0$. Substituting Eqs. (53)-(55) into Eq. (51), and solving the resulting system of algebraic equations, we obtain

$$
\begin{gathered}
\mu_{2}=\mu_{2}, \quad \mu_{3}=\mu_{3}, \quad \tau_{0}=\tau_{0}, \quad \tau_{1}=\tau_{1}, \\
\mu_{0}=\frac{\tau_{0}^{2}\left(\mu_{2} \tau_{1}-2 \mu_{3} \tau_{0}\right)}{\tau_{1}^{3}}, \quad \mu_{1}=\frac{\tau_{0}\left(2 \mu_{2} \tau_{1}-3 \mu_{3} \tau_{0}\right)}{\tau_{1}^{2}}, \\
\omega=\frac{\mu_{2} \tau_{1}(c+\kappa(\sigma-\lambda))-\mu_{3}\left[\kappa(1+n)(\alpha+a \kappa)+3 \tau_{0}(c+\kappa(\sigma-\lambda))\right]}{\mu_{3}(1+n)(1+b \kappa)}, \\
\chi_{0}=-\frac{b v \mu_{2} \tau_{1}(c+\kappa(\sigma-\lambda))-\mu_{3}\left[(1+n)\left(a+b \kappa(a+v \alpha)+a b v \kappa^{2}\right)+3 b v \tau_{0}(c-\kappa \lambda+\kappa \sigma)\right]}{4 n^{2} \tau_{1}(1+b \kappa)(c+\kappa(\sigma-\lambda))} .
\end{gathered}
$$

Substituting these results into Eqs. (18) and (21), we find that

$$
\pm\left(\xi-\xi_{0}\right)=\sqrt{W_{1}} \int \frac{d \Psi}{\sqrt{\Lambda(\Psi)}}
$$

where

$$
\Lambda(\Psi)=\Psi^{3}+\frac{\mu_{2}}{\mu_{3}} \Psi^{2}+\frac{\mu_{1}}{\mu_{3}} \Psi+\frac{\mu_{0}}{\mu_{3}}, \quad W_{1}=\frac{\chi_{0}}{\mu_{3}} .
$$

Consequently, one recovers the traveling wave solutions to the perturbed SHE with STD (46) as follows:

When $\Lambda(\Psi)=\left(\Psi-\lambda_{1}\right)^{3}$, plane wave solution is:

$$
\begin{aligned}
q(x, t)= & \left\{\tau_{0}+\tau_{1} \lambda_{1}+\frac{4 \tau_{1} W_{1}}{\left[x+\left(\frac{\alpha+2 a \kappa+b \omega^{2}}{1+b \omega \kappa}\right) t-\xi_{0}\right]^{2}}\right\}^{\frac{1}{2 n}} \\
& \times \exp \left[i\left\{-\kappa x+\left(\frac{\mu_{2} \tau_{1}(c+\kappa(\sigma-\lambda))-\mu_{3}\left[\kappa(1+n)(\alpha+a \kappa)+3 \tau_{0}(c+\kappa(\sigma-\lambda))\right]}{\mu_{3}(1+n)(1+b \kappa)}\right) t+\theta\right\}\right] .
\end{aligned}
$$

When $\Lambda(\Psi)=\left(\Psi-\lambda_{1}\right)^{2}\left(\Psi-\lambda_{2}\right)$ and $\lambda_{2}>\lambda_{1}$, solitary wave solution is:

$$
\begin{aligned}
q(x, t)= & \left\{\tau_{0}+\tau_{1} \lambda_{2}+\tau_{1}\left(\lambda_{1}-\lambda_{2}\right) \tanh ^{2}\left(\frac{1}{2} \sqrt{\frac{\lambda_{1}-\lambda_{2}}{W_{1}}}\left[x+\left(\frac{\alpha+2 a \kappa+b \omega^{2}}{1+b \omega \kappa}\right) t-\xi_{0}\right]\right)\right\}^{\frac{1}{2 n}} \\
& \times \exp \left[i\left\{-\kappa x+\left(\frac{\mu_{2} \tau_{1}(c+\kappa(\sigma-\lambda))-\mu_{3}\left[\kappa(1+n)(\alpha+a \kappa)+3 \tau_{0}(c+\kappa(\sigma-\lambda))\right]}{\mu_{3}(1+n)(1+b \kappa)}\right) t+\theta\right\}\right] .
\end{aligned}
$$


When $\Lambda(\Psi)=\left(\Psi-\lambda_{1}\right)\left(\Psi-\lambda_{2}\right)^{2}$ and $\lambda_{1}>\lambda_{2}$, hyperbolic function solution is:

$$
\begin{aligned}
q(x, t)= & \left\{\tau_{0}+\tau_{1} \lambda_{1}+\tau_{1}\left(\lambda_{1}-\lambda_{2}\right) \operatorname{cosech}^{2}\left(\frac{1}{2} \sqrt{\frac{\lambda_{1}-\lambda_{2}}{W_{1}}}\left[x+\left(\frac{\alpha+2 a \kappa+b \omega^{2}}{1+b \omega \kappa}\right) t\right]\right)\right\}^{\frac{1}{2 n}} \\
& \times \exp \left[i\left\{-\kappa x+\left(\frac{\mu_{2} \tau_{1}(c+\kappa(\sigma-\lambda))-\mu_{3}\left[\kappa(1+n)(\alpha+a \kappa)+3 \tau_{0}(c+\kappa(\sigma-\lambda))\right]}{\mu_{3}(1+n)(1+b \kappa)}\right) t+\theta\right\}\right] .
\end{aligned}
$$

When $\Lambda(\Psi)=\left(\Psi-\lambda_{1}\right)\left(\Psi-\lambda_{2}\right)\left(\Psi-\lambda_{3}\right)$ and $\lambda_{1}>\lambda_{2}>\lambda_{3}$, Jacobi elliptic function solutions are:

$$
\begin{aligned}
q(x, t)= & \left\{\tau_{0}+\tau_{1} \lambda_{3}+\tau_{1}\left(\lambda_{2}-\lambda_{3}\right) \operatorname{sn}^{2}\left(\mp \frac{1}{2} \sqrt{\frac{\lambda_{1}-\lambda_{3}}{W_{1}}}\left[x+\left(\frac{\alpha+2 a \kappa+b \omega^{2}}{1+b \omega \kappa}\right) t-\xi_{0}\right], l\right)\right\}^{\frac{1}{2 n}} \\
& \times \exp \left[i\left\{-\kappa x+\left(\frac{\mu_{2} \tau_{1}(c+\kappa(\sigma-\lambda))-\mu_{3}\left[\kappa(1+n)(\alpha+a \kappa)+3 \tau_{0}(c+\kappa(\sigma-\lambda))\right]}{\mu_{3}(1+n)(1+b \kappa)}\right) t+\theta\right\}\right],
\end{aligned}
$$

where

$$
l^{2}=\frac{\lambda_{2}-\lambda_{3}}{\lambda_{1}-\lambda_{3}}
$$

Notice $\lambda_{i}(i=1,2,3)$ are the roots of the polynomial equation

$$
\Lambda(\Psi)=0 .
$$

When $\tau_{0}=-\tau_{1} \lambda_{1}$ and $\xi_{0}=0$, solutions (59)-(61) can be reduced to rational function solution

$$
\begin{aligned}
q(x, t)= & \left\{\frac{A_{2}}{x+\left(\frac{\alpha+2 a \kappa+b \omega^{2}}{1+b \omega \kappa}\right) t}\right\}^{\frac{1}{n}} \\
& \times \exp \left[i\left\{-\kappa x+\left(\frac{\mu_{2} \tau_{1}(c+\kappa(\sigma-\lambda))-\mu_{3}\left[\kappa(1+n)(\alpha+a \kappa)+3 \tau_{0}(c+\kappa(\sigma-\lambda))\right]}{\mu_{3}(1+n)(1+b \kappa)}\right) t+\theta\right\}\right],
\end{aligned}
$$

1-soliton solution

$$
\begin{aligned}
q(x, t)= & \left\{\frac{A_{3}}{\cosh ^{\frac{1}{n}}\left[B_{3}\left(x+\left(\frac{\alpha+2 a \kappa+b \omega^{2}}{1+b \omega \kappa}\right) t\right)\right]}\right\} \\
& \times \exp \left[i\left\{-\kappa x+\left(\frac{\mu_{2} \tau_{1}(c+\kappa(\sigma-\lambda))-\mu_{3}\left[\kappa(1+n)(\alpha+a \kappa)+3 \tau_{0}(c+\kappa(\sigma-\lambda))\right]}{\mu_{3}(1+n)(1+b \kappa)}\right) t+\theta\right\}\right],
\end{aligned}
$$

and singular soliton solution

$$
\begin{aligned}
q(x, t)= & \left\{\frac{A_{4}}{\sinh ^{\frac{1}{n}}\left[B_{3}\left(x+\left(\frac{\alpha+2 a \kappa+b \omega^{2}}{1+b \omega \kappa}\right) t\right)\right]}\right\} \\
& \times \exp \left[i\left\{-\kappa x+\left(\frac{\mu_{2} \tau_{1}(c+\kappa(\sigma-\lambda))-\mu_{3}\left[\kappa(1+n)(\alpha+a \kappa)+3 \tau_{0}(c+\kappa(\sigma-\lambda))\right]}{\mu_{3}(1+n)(1+b \kappa)}\right) t+\theta\right\}\right],
\end{aligned}
$$

where

$$
A_{2}=2 \sqrt{\tau_{1} W_{1}}, \quad A_{3}=\left[\tau_{1}\left(\lambda_{2}-\lambda_{1}\right)\right]^{\frac{1}{2 n}}, \quad A_{4}=\left[\tau_{1}\left(\lambda_{1}-\lambda_{2}\right)\right]^{\frac{1}{2 n}}, \quad B_{3}=\frac{1}{2} \sqrt{\frac{\lambda_{1}-\lambda_{2}}{W_{1}}} .
$$


Here, $A_{3}$ and $A_{4}$ are respectively the amplitudes of 1-soliton and singular soliton, while $B_{3}$ is the inverse width of the solitons. These solitons exist for $\tau_{1}>0$. Moreover, when $\tau_{0}=-\tau_{1} \lambda_{3}$ and $\xi_{0}=0$, the solution (62) can be simplified as

$$
\begin{aligned}
q(x, t)= & A_{5} \operatorname{sn}^{\frac{1}{n}}\left(B_{j}\left[x+\left(\frac{\alpha+2 a \kappa+b \omega^{2}}{1+b \omega \kappa}\right) t\right], \frac{\lambda_{2}-\lambda_{3}}{\lambda_{1}-\lambda_{3}}\right) \\
& \times \exp \left[i\left\{-\kappa x+\left(\frac{\mu_{2} \tau_{1}(c+\kappa(\sigma-\lambda))-\mu_{3}\left[\kappa(1+n)(\alpha+a \kappa)+3 \tau_{0}(c+\kappa(\sigma-\lambda))\right]}{\mu_{3}(1+n)(1+b \kappa)}\right) t+\theta\right\}\right],
\end{aligned}
$$

where

$$
A_{5}=\left[\tau_{1}\left(\lambda_{2}-\lambda_{3}\right)\right]^{\frac{1}{2 n}}, \quad B_{j}=\frac{(-1)^{j}}{2} \sqrt{\frac{\lambda_{1}-\lambda_{3}}{W_{1}}}, \quad(j=4,5) .
$$

Remark-3: When the modulus $l \rightarrow 1$, dark soliton solutions emerge as

$$
\begin{aligned}
q(x, t)= & A_{5} \tanh ^{\frac{1}{n}}\left[B_{j}\left(x+\left(\frac{\alpha+2 a \kappa+b \omega^{2}}{1+b \omega \kappa}\right) t\right)\right] \\
& \times \exp \left[i\left\{-\kappa x+\left(\frac{\mu_{2} \tau_{1}(c+\kappa(\sigma-\lambda))-\mu_{3}\left[\kappa(1+n)(\alpha+a \kappa)+3 \tau_{0}(c+\kappa(\sigma-\lambda))\right]}{\mu_{3}(1+n)(1+b \kappa)}\right) t+\theta\right\}\right],
\end{aligned}
$$

where $\lambda_{1}=\lambda_{2}$.

Case-2: When $\varrho=4, \rho=0$ and $\varsigma=2$ in Eq. (52), we have

$$
\begin{gathered}
U=\tau_{0}+\tau_{1} \Psi+\tau_{2} \Psi^{2}, \\
\left(U^{\prime}\right)^{2}=\frac{\left(\tau_{1}+2 \tau_{2} \Psi\right)^{2}\left(\mu_{4} \Psi^{4}+\mu_{3} \Psi^{3}+\mu_{2} \Psi^{2}+\mu_{1} \Psi+\mu_{0}\right)}{\chi_{0}}, \\
U^{\prime \prime}=\frac{\left(\tau_{1}+2 \tau_{2} \Psi\right)\left(4 \mu_{4} \Psi^{3}+3 \mu_{3} \Psi^{2}+2 \mu_{2} \Psi+\mu_{1}\right)+4 \tau_{2}\left(\mu_{4} \Psi^{4}+\mu_{3} \Psi^{3}+\mu_{2} \Psi^{2}+\mu_{1} \Psi+\mu_{0}\right)}{2 \chi_{0}},
\end{gathered}
$$

where $\mu_{4} \neq 0$ and $\chi_{0} \neq 0$. Substituting Eqs. (72)-(74) into Eq. (51), and solving the resulting system of algebraic equations, we find the following results:

$$
\begin{aligned}
& \mu_{0}=\frac{4 n^{2} \tau_{0}^{2} \chi_{0}(1+b \kappa)(c+\kappa(\sigma-\lambda))}{-b v \tau_{1}^{2}(c+\kappa(\sigma-\lambda))+4 \tau_{2}\left[(1+n)\left(a+b \kappa(a+v \alpha)+a b v \kappa^{2}\right)+b v \tau_{0}(c-\kappa \lambda+\kappa \sigma)\right]}, \\
& \mu_{1}=\frac{8 n^{2} \tau_{0} \tau_{1} \chi_{0}(1+b \kappa)(c+\kappa(\sigma-\lambda))}{-b v \tau_{1}^{2}(c+\kappa(\sigma-\lambda))+4 \tau_{2}\left[(1+n)\left(a+b \kappa(a+v \alpha)+a b v \kappa^{2}\right)+b v \tau_{0}(c-\kappa \lambda+\kappa \sigma)\right]}, \\
& \mu_{2}=\frac{4 n^{2} \chi_{0}(1+b \kappa)(c+\kappa(\sigma-\lambda))\left(\tau_{1}^{2}+2 \tau_{0} \tau_{2}\right)}{-b v \tau_{1}^{2}(c+\kappa(\sigma-\lambda))+4 \tau_{2}\left[(1+n)\left(a+b \kappa(a+v \alpha)+a b v \kappa^{2}\right)+b v \tau_{0}(c-\kappa \lambda+\kappa \sigma)\right]}, \\
& \mu_{3}=\frac{8 n^{2} \tau_{1} \tau_{2} \chi_{0}(1+b \kappa)(c+\kappa(\sigma-\lambda))}{-b v \tau_{1}^{2}(c+\kappa(\sigma-\lambda))+4 \tau_{2}\left[(1+n)\left(a+b \kappa(a+v \alpha)+a b v \kappa^{2}\right)+b v \tau_{0}(c-\kappa \lambda+\kappa \sigma)\right]}, \\
& \mu_{4}=\frac{4 n^{2} \tau_{2}^{2} \chi_{0}(1+b \kappa)(c+\kappa(\sigma-\lambda))}{-b v \tau_{1}^{2}(c+\kappa(\sigma-\lambda))+4 \tau_{2}\left[(1+n)\left(a+b \kappa(a+v \alpha)+a b v \kappa^{2}\right)+b v \tau_{0}(c-\kappa \lambda+\kappa \sigma)\right]}, \\
& \omega=\frac{\tau_{1}^{2}(c+\kappa(\sigma-\lambda))-4 \tau_{2}\left[\kappa(1+n)(\alpha+a \kappa)+\tau_{0}(c-\kappa \lambda+\kappa \sigma)\right]}{4 \tau_{2}(1+n)(1+b \kappa)}, \\
& \chi_{0}=\chi_{0}, \quad \tau_{0}=\tau_{0}, \quad \tau_{1}=\tau_{1}, \quad \tau_{2}=\tau_{2} .
\end{aligned}
$$

Substituting these results into Eqs. (18) and (21), we find that

$$
\pm\left(\xi-\xi_{0}\right)=W_{2} \int \frac{d \Psi}{\sqrt{\Lambda(\Psi)}},
$$


where

$$
\Lambda(\Psi)=\Psi^{4}+\frac{\mu_{3}}{\mu_{4}} \Psi^{3}+\frac{\mu_{2}}{\mu_{4}} \Psi^{2}+\frac{\mu_{1}}{\mu_{4}} \Psi+\frac{\mu_{0}}{\mu_{4}}, \quad W_{2}=\sqrt{\frac{\chi_{0}}{\mu_{4}}} .
$$

Integrating Eq. (76) and taking $\xi_{0}=0$, one obtains the traveling wave solutions to the perturbed SHE with STD (46) in the forms:

When $\Lambda(\Psi)=\left(\Psi-\lambda_{1}\right)^{4}$, we obtain

$$
\begin{aligned}
q(x, t)= & {\left[\sum_{j=0}^{2} \tau_{j}\left(\lambda_{1} \pm \frac{W_{2}}{x+\left(\frac{\alpha+2 a \kappa+b \omega^{2}}{1+b \omega \kappa}\right) t}\right)^{j}\right]^{\frac{1}{2 n}} } \\
& \times \exp \left[i\left\{-\kappa x+\left(\frac{\tau_{1}^{2}(c+\kappa(\sigma-\lambda))-4 \tau_{2}\left[\kappa(1+n)(\alpha+a \kappa)+\tau_{0}(c-\kappa \lambda+\kappa \sigma)\right]}{4 \tau_{2}(1+n)(1+b \kappa)}\right) t+\theta\right\}\right] .
\end{aligned}
$$

When $\Lambda(\Psi)=\left(\Psi-\lambda_{1}\right)^{3}\left(\Psi-\lambda_{2}\right)$ and $\lambda_{2}>\lambda_{1}$, we get

$$
\begin{aligned}
q(x, t)= & {\left[\sum_{j=0}^{2} \tau_{j}\left(\lambda_{1}+\frac{4 W_{2}^{2}\left(\lambda_{2}-\lambda_{1}\right)}{4 W_{2}^{2}-\left[\left(\lambda_{1}-\lambda_{2}\right)\left(x+\left(\frac{\alpha+2 a \kappa+b \omega^{2}}{1+b \omega \kappa}\right) t\right)\right]^{2}}\right)^{j}\right]^{\frac{1}{2 n}} } \\
& \times \exp \left[i\left\{-\kappa x+\left(\frac{\tau_{1}^{2}(c+\kappa(\sigma-\lambda))-4 \tau_{2}\left[\kappa(1+n)(\alpha+a \kappa)+\tau_{0}(c-\kappa \lambda+\kappa \sigma)\right]}{4 \tau_{2}(1+n)(1+b \kappa)}\right) t+\theta\right\}\right] .
\end{aligned}
$$

When $\Lambda(\Psi)=\left(\Psi-\lambda_{1}\right)^{2}\left(\Psi-\lambda_{2}\right)^{2}$, we have

$$
\begin{aligned}
q(x, t)= & {\left[\sum_{j=0}^{2} \tau_{j}\left(\lambda_{2}+\frac{\lambda_{2}-\lambda_{1}}{\exp \left[\frac{\lambda_{1}-\lambda_{2}}{W_{2}}\left(x+\left(\frac{\alpha+2 a \kappa+b \omega^{2}}{1+b \omega \kappa}\right) t\right)\right]-1}\right)^{j}\right]^{\frac{1}{2 n}} } \\
& \times \exp \left[i\left\{-\kappa x+\left(\frac{\tau_{1}^{2}(c+\kappa(\sigma-\lambda))-4 \tau_{2}\left[\kappa(1+n)(\alpha+a \kappa)+\tau_{0}(c-\kappa \lambda+\kappa \sigma)\right]}{4 \tau_{2}(1+n)(1+b \kappa)}\right) t+\theta\right\}\right],
\end{aligned}
$$

and

$$
\begin{aligned}
q(x, t)= & {\left[\sum_{j=0}^{2} \tau_{j}\left(\lambda_{1}+\frac{\lambda_{1}-\lambda_{2}}{\exp \left[\frac{\lambda_{1}-\lambda_{2}}{W_{2}}\left(x+\left(\frac{\alpha+2 a \kappa+b \omega^{2}}{1+b \omega \kappa}\right) t\right)\right]-1}\right)^{j}\right]^{\frac{1}{2 n}} } \\
& \times \exp \left[i\left\{-\kappa x+\left(\frac{\tau_{1}^{2}(c+\kappa(\sigma-\lambda))-4 \tau_{2}\left[\kappa(1+n)(\alpha+a \kappa)+\tau_{0}(c-\kappa \lambda+\kappa \sigma)\right]}{4 \tau_{2}(1+n)(1+b \kappa)}\right) t+\theta\right\}\right] .
\end{aligned}
$$

When $\Lambda(\Psi)=\left(\Psi-\lambda_{1}\right)^{2}\left(\Psi-\lambda_{2}\right)\left(\Psi-\lambda_{3}\right)$ and $\lambda_{1}>\lambda_{2}>\lambda_{3}$, we recover

$$
\begin{aligned}
q(x, t)= & {\left[\sum_{j=0}^{2} \tau_{j}\left(\lambda_{1}-\frac{2\left(\lambda_{1}-\lambda_{2}\right)\left(\lambda_{1}-\lambda_{3}\right)}{2 \lambda_{1}-\lambda_{2}-\lambda_{3}+\left(\lambda_{3}-\lambda_{2}\right) \cosh \left[\frac{\sqrt{\left(\lambda_{1}-\lambda_{2}\right)\left(\lambda_{1}-\lambda_{3}\right)}}{W_{2}}\left(x+\left(\frac{\alpha+2 a \kappa+b \omega^{2}}{1+b \omega \kappa}\right) t\right)\right]}\right)^{j}\right]^{\frac{1}{2 n}} } \\
& \times \exp \left[i\left\{-\kappa x+\left(\frac{\tau_{1}^{2}(c+\kappa(\sigma-\lambda))-4 \tau_{2}\left[\kappa(1+n)(\alpha+a \kappa)+\tau_{0}(c-\kappa \lambda+\kappa \sigma)\right]}{4 \tau_{2}(1+n)(1+b \kappa)}\right) t+\theta\right\}\right] .
\end{aligned}
$$


When $\Lambda(\Psi)=\left(\Psi-\lambda_{1}\right)\left(\Psi-\lambda_{2}\right)\left(\Psi-\lambda_{3}\right)\left(\Psi-\lambda_{4}\right)$ and $\lambda_{1}>\lambda_{2}>\lambda_{3}>\lambda_{4}$, we find

$$
\begin{aligned}
q(x, t)= & {\left[\sum_{j=0}^{2} \tau_{j}\left(\lambda_{2}+\frac{\left(\lambda_{1}-\lambda_{2}\right)\left(\lambda_{4}-\lambda_{2}\right)}{\lambda_{4}-\lambda_{2}+\left(\lambda_{1}-\lambda_{4}\right) \mathrm{sn}^{2}\left[ \pm \frac{\sqrt{\left(\lambda_{1}-\lambda_{3}\right)\left(\lambda_{2}-\lambda_{4}\right)}}{2 W_{2}}\left(x+\left(\frac{\alpha+2 a \kappa+b \omega^{2}}{1+b \omega \kappa}\right) t\right), l\right]}\right]^{j}\right]^{\frac{1}{2 n}} } \\
& \times \exp \left[i\left\{-\kappa x+\left(\frac{\tau_{1}^{2}(c+\kappa(\sigma-\lambda))-4 \tau_{2}\left[\kappa(1+n)(\alpha+a \kappa)+\tau_{0}(c-\kappa \lambda+\kappa \sigma)\right]}{4 \tau_{2}(1+n)(1+b \kappa)}\right) t+\theta\right\}\right]
\end{aligned}
$$

where

$$
l^{2}=\frac{\left(\lambda_{2}-\lambda_{3}\right)\left(\lambda_{1}-\lambda_{4}\right)}{\left(\lambda_{1}-\lambda_{3}\right)\left(\lambda_{2}-\lambda_{4}\right)}
$$

Notice $\lambda_{i}(i=1, \ldots, 4)$ are the roots of the polynomial equation

$$
\Lambda(\Psi)=0
$$

Remark-4: When the modulus $l \rightarrow 1$, the hyperbolic function solutions fall out:

$$
\begin{aligned}
q(x, t)= & {\left[\sum_{j=0}^{2} \tau_{j}\left(\lambda_{2}+\frac{\left(\lambda_{1}-\lambda_{2}\right)\left(\lambda_{4}-\lambda_{2}\right)}{\lambda_{4}-\lambda_{2}+\left(\lambda_{1}-\lambda_{4}\right) \tanh ^{2}\left[ \pm \frac{\sqrt{\left(\lambda_{1}-\lambda_{3}\right)\left(\lambda_{2}-\lambda_{4}\right)}}{2 W_{2}}\left(x+\left(\frac{\alpha+2 a \kappa+b \omega^{2}}{1+b \omega \kappa}\right) t\right)\right]}\right]^{j}\right]^{\frac{1}{2 n}} } \\
& \times \exp \left[i\left\{-\kappa x+\left(\frac{\tau_{1}^{2}(c+\kappa(\sigma-\lambda))-4 \tau_{2}\left[\kappa(1+n)(\alpha+a \kappa)+\tau_{0}(c-\kappa \lambda+\kappa \sigma)\right]}{4 \tau_{2}(1+n)(1+b \kappa)}\right) t+\theta\right\}\right]
\end{aligned}
$$

where $\lambda_{3}=\lambda_{4}$.

Remark-5: However, if $l \rightarrow 0$, the periodic wave solution are listed as below:

$$
\begin{aligned}
q(x, t)= & {\left[\sum_{j=0}^{2} \tau_{j}\left(\lambda_{2}+\frac{\left(\lambda_{1}-\lambda_{2}\right)\left(\lambda_{4}-\lambda_{2}\right)}{\lambda_{4}-\lambda_{2}+\left(\lambda_{1}-\lambda_{4}\right) \sin ^{2}\left[ \pm \frac{\sqrt{\left(\lambda_{1}-\lambda_{3}\right)\left(\lambda_{2}-\lambda_{4}\right)}}{2 W_{2}}\left(x+\left(\frac{\alpha+2 a \kappa+b \omega^{2}}{1+b \omega \kappa}\right) t\right)\right]}\right]^{j}\right]^{\frac{1}{2 n}} } \\
& \times \exp \left[i\left\{-\kappa x+\left(\frac{\tau_{1}^{2}(c+\kappa(\sigma-\lambda))-4 \tau_{2}\left[\kappa(1+n)(\alpha+a \kappa)+\tau_{0}(c-\kappa \lambda+\kappa \sigma)\right]}{4 \tau_{2}(1+n)(1+b \kappa)}\right) t+\theta\right\}\right]
\end{aligned}
$$

where $\lambda_{2}=\lambda_{3}$.

\section{CONCLUSIONS}

This paper employed the extended trial equation method to secure bright, dark and singular optical soliton solutions to SHE. In addition plane waves, periodic waves and other solutions emerged as a byproduct. Both Kerr law and power law are studied. The results of this paper provides basic foundation to further research in the avenue of dispersive optical solitons. Birefringent fibers, DWDM systems and metamaterials will be later touched upon. While the perturbation terms in this paper are considered to be strong, quasi-stationary soliton solutions can be obtained for weak perturbations. These results will be reported in future. Additional integration tools will be further implemented to reecover more interesting solutions that are awaited at this time. 


\section{ACKNOWLEDGEMENTS}

The sixth author (QZ) was funded by the National Science Foundation of Hubei Province in China under the grant number 2015CFC891. The seventh author (SPM) would like to thank the research support provided by the Department of Mathematics and Statistics at Tshwane University of Technology and the support from the South African National Foundation under Grant Number 92052 IRF1202210126. The eighth author (AB) would like to thank Tshwane University of Technology during his academic visit during 2016. The research work of ninth author (MB) was supported by Qatar National Research Fund (QNRF) under the grant number NPRP 6-021-1-005. The authors also declare that there is no conflict of interest. 


\section{References}

[1] A. Hasegawa, Y. Kodama, Solitons in Optical Communications. Oxford University Press. Oxford, UK. (1995).

[2] Y. Kodama, A. Hasegawa, IEEE Journal of Quantum Electronics, QE-23, 510 (1987).

[3] S. Wabnitz, Y. Kodama, A. B. Aceves, Optical Fiber Technology 1, 187 (1995).

[4] X. Geng, Y. Lv, Nonlinear Dynamics 69, 1621 (2012).

[5] S. Kumar, K. Singh, R. K. Gupta, Pramana 79, 41 (2012).

[6] C. S. Liu, Communications in Theoretical Physics 49, 153 (2008).

[7] C. S. Liu, Computer Physics Communications 181, 317 (2010).

[8] M. Ekici, M. Mirzazadeh, and M. Eslami, Nonlinear Dynamics 84, 669 (2016).

[9] Q. Zhou, M. Ekici, A. Sonmezoglu, M. Mirzazadeh, and M. Eslami, Nonlinear Dynamics 84, 1883 (2016).

[10] Y. Gurefe, E. Misirli, A. Sonmezoglu, and M. Ekici, Applied Mathematics and Computation 219, 5253 (2013).

[11] A. H. Bhrawy, A. A. Alshaery, E. M. Hilal, W. Manrakhan, M. Savescu, A. Biswas, Journal of Nonlinear Optical Physics and Materials 23, 1450014 (2014).

[12] A. Biswas, A. J. M. Jawad, W. N. Manrakhan, A. K. Sarma, K. R. Khan, Optics \& Laser Technology 44, 2265 (2012).

[13] R. Kohl, A. Biswas, D. Milovic, E. Zerrad, Optics \& Laser Technology 40, 647 (2008).

[14] M. Mirzazadeh, M. Ekici, A. Sonomezoglu, Q. Zhou, H. Triki, S. P. Moshokoa, A. Biswas \&b M. Belic. "Optical solitons in birefringent fibers by extended trial equation method". Optik. Volume 127, Issue 23, 11311-11325. (2016).

[15] A. H. Arnous, M. Mirzazadeh, Q. Zhou, S. P. Moshokoa, A. Biswas \& M. Belic. "Optical solitons with higher order dispersions in parabolic law medium by trial solution approach". Optik. Volume 127, Issue 23, 11306-11310. (2016). 